\title{
INVESTIMENTOS ESTADUAIS PÚBLICOS E PRIVADOS: "BENS" SUBSTITUTOS OU COMPLEMENTARES?
}

\author{
Nathalie Gimenes Sanches * \\ Fabiana Rocha ${ }^{\dagger}$
}

\begin{abstract}
Resumo
O objetivo deste artigo é quantificar a relação entre os investimentos público e privado durante o período 1991-2004. Os resultados obtidos confirmam, para o Brasil e seus Estados, a existência de uma forte relação de complementaridade entre os dois tipos de investimento, sendo que as estimativas variam entre $(0,740)$ e $(1,135)$. Somente no caso dos estados de Alagoas, Goiás, Mato Grosso, Pernambuco e Sergipe as elasticidades são relativamente baixas, variando entre $(0,447)$ e $(0,668)$.
\end{abstract}

Palavras-chave: Investimento público; Investimento privado; Modelos de dados em painel

\begin{abstract}
The purpose of this paper is to quantify the relationship between public and private investment during the period 1991-2004. The results indicate the existence of strength complementarity's relationship between both type of investments, both for Brazil and its States, with the estimative ranging between $(0,740)$ and $(1,135)$. Only for the states of Alagoas, Goiás, Mato Grosso, Pernambuco and Sergipe the elasticity are relatively low, ranging from $(0,447)$ to $(0,668)$.
\end{abstract}

Keywords: Public investment; Private investment; Panel data models. JEL classification: C01, C33, C51, E22, E66.

\section{Introdução}

O Programa de Aceleração do Crescimento, lançado em janeiro de 2007, engloba um conjunto de políticas que tem como propósito acelerar o crescimento da economia brasileira. O investimento em infra-estrutura constitui um dos seus cinco blocos e tem como objetivo explícito estimular o aumento do investimento privado. ${ }^{1}$

\footnotetext{
${ }^{*}$ Mestrando em Economia pela Queen Mary - University of London

+ FEA/USP

${ }^{1}$ Os demais blocos são constituídos pelo estímulo ao crédito e ao financiamento, pela melhora do ambiente de investimento, pela desoneração e aperfeiçoamento do sistema tributário e pelas medidas fiscais de longo prazo.
} 
A racionalidade por trás do programa é a crença de que os gastos em investimento do Governo exercem um efeito positivo sobre os investimentos privados. Esta crença, no entanto, do ponto de vista acadêmico, é objeto de intensa disputa.

Basicamente três argumentos compõem a racionalidade econômica para a complementaridade. O primeiro reside na idéia de que o investimento público é composto primordialmente de bens e serviços que o setor privado não irá fornecer em quantidades ótimas, seja porque é difícil avaliar o seu uso e benefício para os consumidores, seja porque eles estão sujeitos a altos custos de implantação. Esses bens públicos, contudo, são fundamentais para o funcionamento do mercado uma vez que trazem consigo grandes efeitos de transbordamento. O segundo tem como fundamento o efeito indireto do investimento público sobre a formação de capital privado, na medida em que ele aumenta a demanda agregada corrente por bens e serviços produzidos pelo setor privado, assim como as perspectivas de vendas futuras. O terceiro assume que o investimento público em infra-estrutura aumenta a produtividade total dos fatores assim como a produtividade total do trabalho.

A racionalidade econômica básica para a substitutibilidade reside no argumento de que o investimento público financiado de forma subsidiada e levado adiante por empresas estatais ineficientes acaba reduzindo as possibilidades para o investimento privado.

O objetivo deste artigo é investigar a relação existente entre os investimentos público e privado, mais precisamente o impacto do investimento público sobre o privado, a nível estadual e regional. A grande dificuldade neste tipo de análise é a falta de dados sobre o investimento público e o investimento privado em cada Estado. Dessa forma, procura-se construir essas variáveis a partir da participação do investimento de cada Estado no total do investimento em construções no país como sugerido por Silveira (2005).

Este artigo está organizado em quatro seções, além desta introdução. $\mathrm{Na}$ segunda seção, apresenta-se uma breve revisão da evidência empírica existente sobre a relação entre os investimentos público e privado, com o objetivo de apresentar a falta de consenso existente. Na terceira seção, apresentaremos um modelo para quantificar as relações entre esses dois tipos de investimento, considerando na quarta seção um resultado geral para o Brasil e também um específico para cada estado brasileiro. Finalmente, na última seção, apresentaremos a conclusão do trabalho realizado.

\section{Breve discussão da evidência empírica existente}

Vários pesquisadores focalizaram sua atenção na questão de se investimentos públicos e privados são complementares, substitutos ou independentes (como assumido em muitos modelos macroeconômicos).

Aschauer (1989), em um estudo para a economia americana, conclui que expansões nos gastos do governo com investimento público teriam um impacto estimulante maior sobre a produção privada do que se a expansão fosse aos gastos do governo com consumo. Isso porque o investimento público induz um aumento na taxa de retorno do capital privado, o que estimula o gasto privado com investimento.

Easterly \& Rebelo (1993), por outro lado, concluem que para a economia americana o investimento público total e o investimento público das estatais 
são negativamente, e consistentemente, correlacionados com o investimento privado. Analisam também a influência de alguns setores sobre o crescimento, concluindo que: i) investimento em transporte e comunicação é positivamente correlacionado com crescimento; ii) o investimento no setor agrícola gera um efeito negativo sobre o crescimento e sobre o investimento privado; iii) o investimento público em moradias e infra-estrutura urbana é positivamente, e consistentemente, correlacionado com o crescimento e com o investimento privado.

Bajo \& Sosvilla (1993) procuram evidências sobre o efeito da acumulação de capital do setor público sobre a produtividade do setor privado usando dados da Espanha e encontram evidências que dão suporte à visão de que o estoque de capital público tem um importante papel sobre a produtividade do setor privado.

Servén (1996) investiga a relação entre o investimento público e o privado na Índia e percebe a existência de heterogeneidade no capital público, a qual implica que diferentes tipos de investimento público podem ter efeitos opostos sobre as atividades do setor privado, ou seja, efeitos de "crowding in" e "crowding out". Projetos públicos em áreas como infra-estrutura básica e formação de capital humano tendem a aumentar a lucratividade da produção privada e, portanto, encorajam o investimento privado. Projetos públicos em atividades convencionais, em que empresas públicas basicamente replicam as ações de firmas privadas, têm efeito oposto causado pela competição com o setor privado pelo uso de bens e fatores do mercado. Assim, encontra evidência de que um aumento do capital de infra-estrutura pública aumenta a lucratividade da produção privada e também aumenta o estoque de capital privado de longo prazo. Contrariamente, um aumento do capital não relacionado à infra-estrutura tem um efeito ambíguo, ou seja, pode levar a um aumento ou a uma redução do estoque de capital privado, dependendo da elasticidade de substituição entre os bens finais ofertados pelos dois setores, considerando a hipótese de que esses bens finais de ambos os setores sejam substitutos imperfeitos.

Pinho (2001) também define essa relação de "crowding in" e "crowding out" em uma análise sobre o impacto do capital público no crescimento da produtividade da região da Galícia (Espanha). Se o investimento público utilizar recursos limitados e potencializar a produção de bens concorrentes ou substitutos dos bens produzidos pelo setor privado, pode levar a um efeito "crowding out", ou seja, de expulsão do investimento privado. Por outro lado, se o investimento público potencializar a produção de bens complementares aos produzidos pelo setor privado, como no caso de infra-estrutura social e econômica, permitindo um aumento da produtividade marginal do capital privado, o efeito pode ser de "crowding in", ou seja, de complementaridade ao investimento privado.

Em um estudo para o México, Ramirez (1996) conclui que um aumento de $10 \%$ no investimento público gera um aumento entre $2 \%$ a $3 \%$ sobre a formação bruta de capital privado dentro de um ano, ou seja, no curto prazo. Já no longo prazo, a elasticidade do investimento público é de 0,63.

Nazmi \& Ramirez (1997) analisam, também para o México, o impacto do investimento público e do privado sobre o crescimento. Chegam à conclusão de que tanto o investimento público quanto o investimento privado têm um efeito positivo e significante sobre o crescimento, apresentando os dois tipos de investimento um impacto estatisticamente idêntico sobre o crescimento. Já 
com relação ao efeito de um investimento sobre o outro, concluem que há um efeito de substituição entre eles.

A falta de consenso também marca a evidência para a economia brasileira. Sant'Ana et al. (1994) encontram evidências de que há complementaridade entre os dois tipos de investimentos, enquanto Rocha \& Teixeira (1996) encontram evidências de um grau de substituição entre os dois tipos de investimentos.

\section{Metodologia}

O grande limitador de uma análise da relação entre investimento público e privado para a economia brasileira reside na falta de dados para o investimento privado.

Para contornar este problema decidimos por utilizar a metodologia elaborada por Silveira (2005), em sua análise sobre o crescimento regional de longo prazo, na qual a construção dessas variáveis leva em consideração a participação do investimento de cada Estado no total do investimento em construções do país. O investimento em construções é escolhido pela sua importância na determinação da Formação Bruta de Capital Fixo do país (cerca de dois terços do total).

Os componentes da Formação Bruta de Capital Fixo (FBCF) são: máquinas e equipamentos, construção e outros. A não inclusão do investimento em máquinas e equipamentos ocorreu devido à falta de dados ou aproximações que os representassem com confiabilidade. Como o componente "máquinas e equipamentos" representa cerca de $30 \%$ do total e acompanha de forma mais ou menos regular as oscilações apresentadas no componente construções no período de 1970 a 2000, optamos por considerar somente o componente construções para a determinação do investimento público em construções de cada estado.

\subsection{Cálculo para Estimação das Variáveis}

\section{Investimento Total em Construções de Cada Estado}

Para o cálculo do Investimento Total em Construções de cada Estado, utilizamos a participação de cada Estado no Investimento Total em Construções do país, a qual é definida utilizando um indicador do volume de investimento realizado e um índice de preços da construção, ambos representados respectivamente por: participação no consumo aparente de cimento e valor do metro quadrado das construções PersonNameProductIDem cada Unidadeem cada Unidade da Federação. Os dados para esses cálculos são fornecidos pelo Sindicato Nacional das Indústrias de Cimento (SNIC) e pelo Sistema Nacional de Custos e índices da Construção Civil (SINAPI - IBGE). O consumo aparente de cimento é uma alternativa à dificuldade de obtenção de dados para a formação bruta de capital fixo em alguns períodos. Para obtermos a participação do estado j no período t no Investimento Total em Construções do país optamos por fazer essa aproximação, dado que tínhamos disponíveis dados explícitos para cada estado. Essa metodologia também foi adotada por Morandi \& Reis (2004). É possível observar uma relação temporal, considerando análise nacional, entre a FBCF em construções e o consumo aparente de cimento. 
A participação de cada Estado no Investimento Total em Construções do país calculada é então aplicada sobre o Investimento Total em Construções do país, resultando na variável Investimento Total em Construções do Estado $j$ no período $t$. O investimento Total em Construções do país é estimado pelo método de estoque perpétuo aplicado aos dados de estoque bruto de capital fixo - considerando o componente "construções"-, o qual, por sua vez, é estimado a partir dos dados da formação bruta de capital fixo. Portanto, consideramos que esse investimento engloba as obras de construção civil em geral, não somente a construção de prédios, dado que é representado pelo componente construção da FBCF. A a participação do Estado $j$ no investimento em construções do país no período $t$ é

$$
i c_{j t}=\frac{\left(C A C_{j t}\right) \times\left(S I N A P I_{j t}\right)}{\sum_{j}\left(C A C_{j t}\right)\left(S I N A P I_{j t}\right)}
$$

em que SINAPI St $_{t}$ é o custo médio do metro quadrado no Estado $j$ no período $t$, fornecido pelo Sistema Nacional de Preços e Custos da Indústria da Construção; $C A C_{j t}$ é o consumo aparente de cimento do Estado $j$ no período $t$; Dados fornecidos pelo SNIC (Sindicato Nacional da indústria do Cimento).

$$
I C_{j t}=I C_{t} \times i c_{j t}
$$

ICjt = investimento total em construções do Estado $\mathrm{j}$ no período $\mathrm{t}$;

icjt = participação do Estado $\mathrm{j}$ no investimento em construções do país no período t;

ICt $=$ investimento total em construções do país no período t;

$$
I C_{t}=K_{t+1}-(1-\delta) K_{t}
$$

$\mathrm{Kt}+1$ e Kt = capital fixo - estoque bruto - construção $\mathrm{R} \$$ de 1999 (bilhões) - IPEA. Fonte: Morandi e Reis - "Estoque de capital fixo no Brasil - 19502002". XXXII Encontro Nacional de Economia (2004);

$\delta=$ taxa de depreciação (6\%), utilizada por Ferreira e Malliagros (1998).

\section{Investimento Público em Construções de Cada Estado}

Já para o cálculo de Investimento em Construções das Administrações Públicas do Estado $j$ no período $t$ (ou seja, o Investimento Público em Construções), optamos por aplicar a participação do Estado $j$ no período $t$ na FBCF total do país sobre o Investimento em Construções das Administrações Públicas do país. Esse Investimento também é estimado pelo método de estoque perpétuo aplicado aos dados de estoque bruto de capital fixo das Administrações Públicas - considerando o componente "construções" -, o qual, por sua vez, é estimado a partir dos dados da formação bruta de capital fixo.

$$
I C_{j t}^{G}=\frac{\left(F B C F_{\text {Constrjt }}\right) \times\left(I C_{t}^{G}\right)}{\sum_{j} F B C F_{\text {Constrjt }}}
$$

Onde:

1. ICGjt = investimento em construções das administrações públicas do estado j no período $t$; 
2. FBCFConstrjt $=$ FBCF em construções do estado $j$ no período $t{ }^{2}$

3. ICGt $=$ investimento em construções das administrações públicas no Brasil no período $t$ :

$$
I C_{t}^{G}=K_{t+1}^{G}-(1-\delta) K_{t}^{G}
$$

$\mathrm{KGt}+1$ e KGt = capital fixo - estoque bruto - construção - adm. públicas R\$ de 1999 (bilhões) - IPEA. Cálculo a partir do método do estoque perpétuo. Fonte: Morandi e Reis (2004).

$$
\begin{aligned}
& \text { Investimento Privado em Construções de Cada Estado } \\
& \qquad I C_{j t}^{E}=I C_{j t}-I C_{j t}^{G}
\end{aligned}
$$

Onde:

1. ICEjt = investimento em construções das empresas e famílias do estado j no período t;

2. $\mathrm{ICjt}=$ investimento total em construções do estado $\mathrm{j}$ no período $\mathrm{t}$;

3. $\mathrm{ICGjt}=$ investimento em construções das administrações públicas do estado j no período $t$.

\section{Modelo Econométrico}

O modelo apresentado abaixo foi elaborado com o objetivo de investigar a influência do investimento público sobre o investimento privado, tanto no caso agregado para o Brasil, como também desagregado por estados.

$$
\ln I C_{j t}^{E}=\beta_{1}+\beta_{2} \ln P I B_{j t}+\beta_{3} \ln I C_{j t}^{G}+\beta_{4} J_{t}+w_{t}
$$

Onde:

1. ICEjt = investimento em construções das empresas e famílias do estado j no período $t$;

2. PIBjt $=$ Produto Interno Bruto do estado $\mathrm{j}$ no período $\mathrm{t}$;

3. $\mathrm{ICGjt}=$ investimento em construções das administrações públicas do estado j no período $t$.

4. Jt = Taxa de Juros Anual no período t.

As variáveis acima estão apresentadas em logaritmo natural a fim de obter diretamente suas elasticidades (com exceção da variável Juros). O modelo segue a forma de um painel e, para efeito de comparação, será estimado tanto por efeito fixo como por efeito aleatório, considerando a inclusão ou não de variáveis instrumentais. As variáveis instrumentais escolhidas são: investimento público de cada estado defasado em um período e PIB de cada estado defasado em um período. O uso dessas variáveis instrumentais é necessário

\footnotetext{
${ }^{2}$ Dados obtidos em Silveira, 2005.
} 
devido ao problema de endogeneidade presente no modelo, já que PIB determina investimento privado e vice-versa. Por conta disso, essas variáveis endógenas são correlacionadas com os erros idiossincráticos, o que leva a estimativas viesadas dos parâmetros. As variáveis instrumentais são consideradas como dadas, não sendo correlacionadas com os erros idiossincráticos, no entanto, apresentam correlacão com as variáveis endógenas. Desta forma, as variáveis instrumentais acabam não tendo nenhum efeito parcial sobre a variável explicada, não sendo também correlacionadas com os fatores não observados (erros idiossincráticos) que afetam a variável explicada.

Como é possível que algumas variáveis omitidas no modelo deixem os coeficientes estimados viesados, é necessário adicionar um efeito fixo não observado, ou seja, controlar por fatores não observados, constantes no tempo, que também afetam a variável dependente. Como $j$ representa estados diferentes, a variável aj é chamada de efeito não observado do estado $j$ ou efeito fixo do estado $j$, representando todos os fatores que afetam o investimento privado do estado $j$ que não mudam ao longo do tempo. É importante considerar que fatores históricos dos estados também são efetivamente capturados pelo efeito não observado $a j$.

$\mathrm{Na}$ estimação por efeito fixo, supõe-se que o efeito não observado, $a j$, seja correlacionado com uma ou mais variável explicativa, sendo que o principal objetivo da estimação é eliminar o efeito fixo do modelo. Entretanto, se aj for não correlacionado com cada variável explicativa em todos os períodos de tempo, então o uso de uma transformação para eliminar esse efeito fixo resultará em estimadores ineficientes. Neste caso, o melhor é efetuar a estimação por efeito aleatório.

Normalmente, a decisão sobre utilizar efeito fixo ou aleatório se dá com base na hipótese de se o efeito não observado é melhor entendido como parâmetro a ser estimado ou como resultado de uma variável aleatória. Quando não podemos considerar as observações como extrações aleatórias, é melhor considerar aj como parâmetro a ser estimado, efetuando neste caso a estimação por efeito fixo. Entretanto, se tratarmos o efeito não observado aj como resultado de uma variável aleatória, temos então que basear a decisão de utilizar a estimação por efeito fixo ou aleatório na existência ou não de correlação entre aj e as variáveis explicativas (Wooldridge 2003). Portanto, a fim de comparar os resultados, optamos por realizar tanto a estimação por efeito fixo como também por efeito aleatório.

Também para efeito de comparação dos resultados, em alguns momentos optamos por incluir dummies estaduais, interagindo com o investimento público. Com isso, procura-se captar o efeito específico em cada estado do investimento público sobre o investimento privado. Também optamos por incluir dummies regionais, representadas pelas macroregiões brasileiras. A necessidade de criar dummies regionais foi devido ao painel com variáveis instrumentais, que torna a estimação inviável quando todas as 27 (vinte e sete) interações estaduais criadas são consideradas como variáveis instrumentais.

As interações estaduais foram criadas a partir da multiplicação da dummy do estado $j$ pelo investimento público do respectivo estado. Já as interações regionais foram criadas a partir da multiplicação da dummy da região k pelo investimento público da mesma região $k$. 


\section{Resultados}

Como já foi dito anteriormente, a fim de comparar os resultados, optamos por estimar o modelo acima em três diferentes tipos de regressões, cada uma de quatro maneiras distintas: painel estimado por efeito aleatório; painel estimado por efeito fixo; painel estimado por efeito aleatório, incluindo variáveis instrumentais; e painel estimado por efeito fixo, incluindo variáveis instrumentais. Nas regressões estimadas, a variável dependente é o investimento privado estadual ou regional, e as variáveis independentes são: PIB (estadual ou regional), investimento público (estadual ou regional) e taxa de juros. Em todos os casos, consideramos uma taxa de depreciação de $6 \%$, o que já foi utilizado por Ferreira \& Malliagros (1998).

Os três conjuntos de regressões estimados procuram investigar os determinantes do investimento privado e, principalmente, o impacto do investimento público sobre o privado. O primeiro conjunto de regressões do modelo efetua a análise através de um painel sem levar em consideração as interações criadas pelas dummies estaduais e regionais. Pelos resultados apresentados na Tabela 1, podemos afirmar que o impacto do PIB sobre o investimento privado é de aproximadamente $(0,751)$, ou seja, um aumento de $1 \%$ no PIB estadual causa um aumento de aproximadamente $0,75 \%$ sobre o investimento privado, sendo este valor altamente significante. Com relação ao investimento público, o resultado varia de $(0,917)$ a $(1,0)$, sendo que um aumento de $1 \%$ no investimento público pode levar a um aumento de $0,92 \%$ a $1 \%$ sobre o investimento privado. Dos quatro resultados obtidos, podemos dizer que três deles são altamente significantes. Somente no caso de painel estimado por efeito fixo, com variáveis instrumentais, o coeficiente não é significante ao nível de $10 \%$ de significância. Já com relação aos juros, os coeficientes obtidos variam de $(-0,005)$ a $(-0,011)$, sendo que um aumento de $1 \%$ na taxa de juros pode levar a uma queda de $0,005 \%$ a $0,011 \%$ sobre o investimento privado, sendo os coeficientes altamente significantes.

Tabela 1: Modelo sem Interações Estaduais (1990-2000)

\begin{tabular}{lcccrr}
\hline \multirow{2}{*}{ Coeficientes: } & \multicolumn{2}{c}{ Painel } & & \multicolumn{2}{c}{$\begin{array}{c}\text { Painel com variáveis } \\
\text { instrumentais }\end{array}$} \\
\cline { 2 - 3 } \cline { 6 - 6 } & $\begin{array}{c}\text { Efeito } \\
\text { fixo }\end{array}$ & $\begin{array}{c}\text { Efeito } \\
\text { aleatório }\end{array}$ & & $\begin{array}{c}\text { Efeito } \\
\text { fixo }\end{array}$ & $\begin{array}{c}\text { Efeito } \\
\text { aleatório }\end{array}$ \\
\hline PIB & 0,751 & 0,053 & & $-0,766$ & $-0,002$ \\
& $(0,000)$ & $(0,007)$ & & $(0,896)$ & $(0,950)$ \\
Investimento público & 0,917 & 0,945 & & $-0,576$ & $-1,001$ \\
& $(0,000)$ & $(0,000)$ & & $(0,843)$ & $(0,000)$ \\
Juros & 0,001 & 0,010 & & $-0,015$ & $-0,011$ \\
& $(0,000)$ & $(0,000)$ & & $(0,667)$ & $(0,950)$ \\
\hline
\end{tabular}

Nota: p-valor entre parênteses

Fonte: Tabela elaborada pelas autoras

O segundo conjunto de regressões do modelo somente considera um painel estimado por efeito fixo e efeito aleatório, sem variáveis instrumentais, incluindo também as interações criadas pelas dummies estaduais.

A partir dos resultados apresentados na tabela 2, podemos afirmar que para a maioria dos estados brasileiros observa-se um alto impacto do investi- 
mento público sobre o investimento privado, sendo que o coeficiente varia de $(0,740)$ a $(1,135)$. Assim, um aumento de $1 \%$ no investimento público pode provocar um aumento de $0,74 \%$ a $1,13 \%$ no investimento privado, sendo que todos esses coeficientes são altamente significantes. O impacto nos estados de Alagoas, Goiás, Mato Grosso, Pernambuco e Sergipe é relativamente baixo em comparação ao impacto nos demais estados. O coeficiente desses estados varia de $(0,477)$ a $(0,668)$, sendo significantes ao nível de $10 \%$. Os Estados do Espírito Santo, Minas Gerais, Pará, Rio de Janeiro, Rio Grande do Sul e São Paulo apresentam coeficientes insignificantes ao nível de $10 \%$, quando o painel é estimado por efeito fixo. Com relação aos juros, os coeficientes obtidos foram mais baixos, variando de $(-0,003)$ a $(-0,005)$, sendo que um aumento de $1 \%$ na taxa de juros pode levar a uma queda de $0,003 \%$ a $0,005 \%$ sobre o investimento privado, sendo os coeficientes altamente significantes.

O terceiro conjunto de regressões do modelo leva em consideração as interações criadas pelas dummies regionais. As regressões também foram estimadas das quatro maneiras distintas anteriores. Pelos resultados apresentados na Tabela 3, podemos dizer que todas as macroregiões brasileiras apresentam um alto impacto do investimento público sobre o privado, apresentando coeficientes que variam de $(0,897)$ a $(0,999)$, ou seja, um aumento de $1 \%$ no investimento público pode gerar um aumento de $0,89 \%$ a $1 \%$ no investimento privado. O único caso que apresenta um coeficiente baixo é o da região Sul quando o painel sem variáveis instrumentais é estimado por efeito fixo, sendo que o coeficiente resultante é $(0,656)$. Todas as regiões apresentam coeficientes insignificantes, ao nível de $10 \%$, quando o painel com variáveis instrumentais é estimado por efeito fixo. Já com relação aos juros, os coeficientes obtidos variam de $(-0,005)$ a $(-0,011)$, sendo que um aumento de $1 \%$ na taxa de juros pode levar a uma queda de $0,005 \%$ a $0,011 \%$ sobre o investimento privado, sendo os coeficientes altamente significantes.

Portanto, a partir dos resultados apresentados acima, podemos concluir que há uma forte evidência de complementaridade entre os investimentos público e privado em construção civil. As estimativas obtidas parecem ser, contudo, extremamente elevadas. No entanto, estão de acordo com as obtidas por Easterly \& Rebelo (1993), para a economia americana, quando é discutida a relação entre os dois investimentos, tanto total quanto separado por setores. Considerando os investimentos totais, o investimento público é negativamente correlacionado com o investimento privado, com o coeficiente variando de $(-0,19)$ a $(-0,24)$. Entretanto, quando é considerado o investimento do setor de moradias e infra-estrutura urbana, o resultado é positivo, ou seja, o investimento público em moradias e infra-estrutura urbana é positivamente, e consistentemente, correlacionado com o investimento privado, o coeficiente variando de $(1,01)$ a $(2,1)$. Considerando o investimento em construções como uma "proxy" dos investimentos em moradias e infra-estrutura urbana, é possível acreditar que esta forte relação entre os dois tipos de investimento seja viável, já que o trabalho de Easterly \& Rebelo (1993) é considerado um clássico da literatura internacional.

Além disso, de acordo com um estudo de Barros (2005) sobre as Parcerias Público-Privadas e suas experiências internacionais, o investimento total em infra-estrutura no Chile praticamente quintuplicou no período de 1990 a 2003 , sendo que após a entrada do investimento privado por meio das parcerias com o setor público, o investimento público quase dobrou. Isso mostra, mais uma vez, portanto, a forte relação entre esses dois tipos de investimento, 


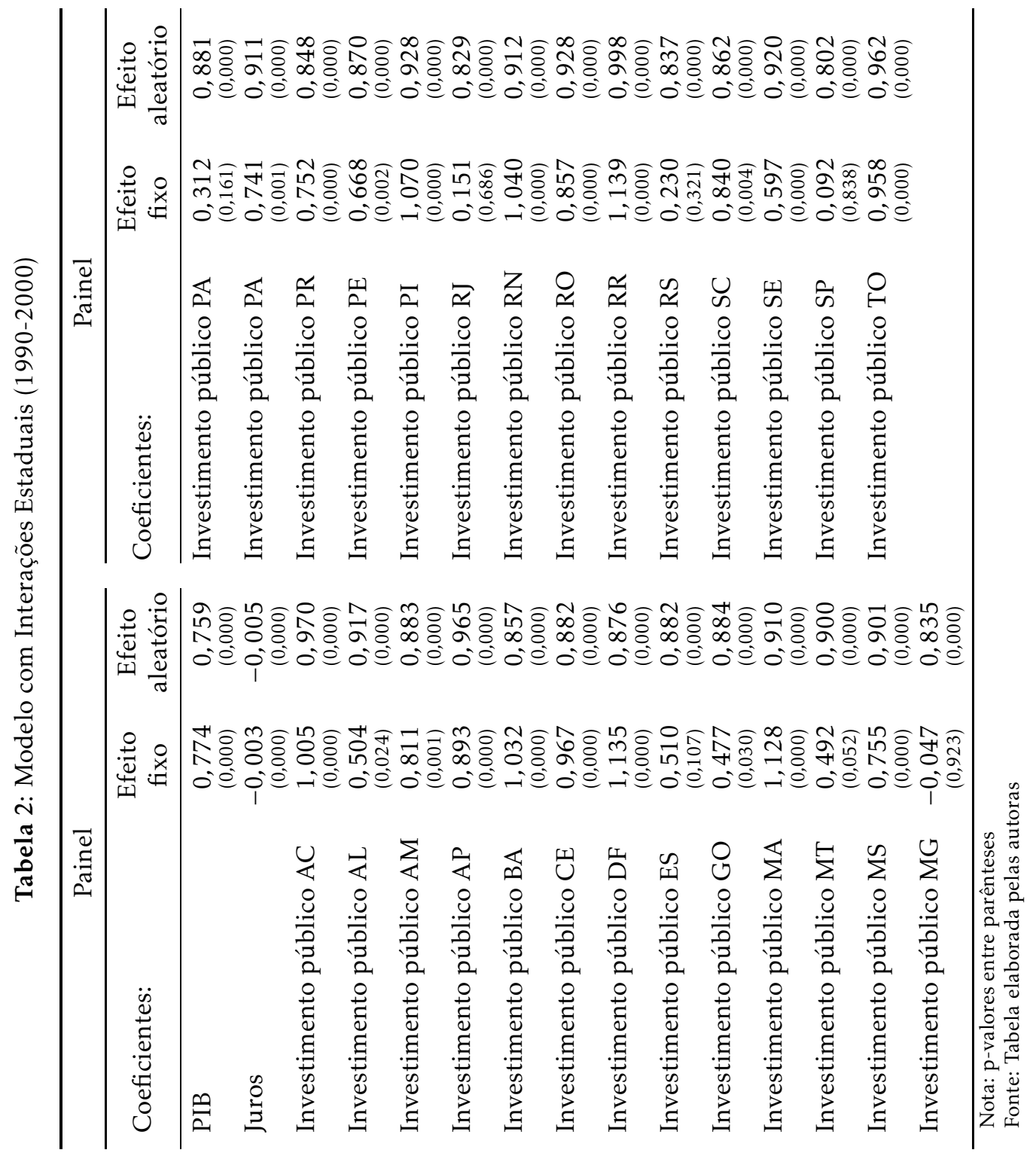


Tabela 3: Modelo com Interações Regionais (1990-2000)

\begin{tabular}{lrrrrr}
\hline & \multicolumn{2}{c}{ Painel } & & $\begin{array}{c}\text { Painel com variáveis } \\
\text { instrumentais }\end{array}$ \\
\cline { 2 - 3 } \cline { 6 - 7 } Coeficientes: & $\begin{array}{c}\text { Efeito } \\
\text { fixo }\end{array}$ & $\begin{array}{c}\text { Efeito } \\
\text { aleatório }\end{array}$ & & $\begin{array}{c}\text { Efeito } \\
\text { fixo }\end{array}$ & $\begin{array}{c}\text { Efeito } \\
\text { aleatório }\end{array}$ \\
\hline PIB & 0,718 & 0,054 & & 1,562 & $-0,001$ \\
& $(0,000)$ & $(0,006)$ & & $(0,300)$ & $(0,959)$ \\
Inv. pub. reg. Norte & 0,953 & 0,947 & & 1,595 & 0,998 \\
& $(0,000)$ & $(0,000)$ & & $(0,523)$ & $(0,000)$ \\
Inv. pub. reg. Nordeste & 0,963 & 0,947 & & 1,013 & 0,999 \\
& $(0,000)$ & $(0,000)$ & & $(0,422)$ & $(0,000)$ \\
Inv. pub. reg. Sudeste & 0,261 & 0,946 & & 1,217 & 0,999 \\
& $(0,206)$ & $(0,000)$ & & $(0,790)$ & $(0,000)$ \\
Inv. pub. reg. Sul & 0,656 & 0,946 & & 5,790 & 0,999 \\
Inv. pub. reg. Centro Oeste & $(0,000)$ & $(0,000)$ & & $(0,669)$ & $(0,000)$ \\
& 0,897 & 0,947 & & 1,614 & 0,999 \\
Juros & $(0,000)$ & $(0,000)$ & $(0,590)$ & $(0,000)$ \\
& $-0,005$ & $-0,010$ & $-0,004$ & $-0,011$ \\
& $(0,000)$ & $(0,000)$ & $(0,631)$ & $(0,000)$ \\
\hline
\end{tabular}

Nota: p-valores entre parênteses

Fonte: Tabela elaborada pelas autoras

sobretudo considerando que o principal objetivo das parcerias é recuperar o investimento público e melhorar a qualidade dos serviços prestados à sociedade.

É possível, portanto, acreditar nessa forte relação de complementaridade entre ambos os investimentos. Entretanto, a análise efetuada restringe-se ao setor de construção civil, o qual está inteiramente relacionado à infraestrutura urbana. Por isso, seria muito construtivo realizar uma análise sobre os investimentos em outros setores, de forma a verificar se essa relação de complementaridade permanece e se também apresenta um alto coeficiente.

\section{Conclusão}

No presente trabalho, foi realizada uma análise quantitativa sobre a relação existente entre os investimentos público e privado, mais precisamente o impacto do investimento público sobre o investimento privado, a nível estadual e regional.

O primeiro conjunto de regressões analisado levou em consideração o caso geral dos estados brasileiros, não tratando da relação específica de cada um destes estados. As elasticidades estimadas variaram de $(0,917)$ a $(1,0)$, ou seja, um aumento de $1 \%$ no investimento público leva a um aumento entre $0,92 \%$ a $1 \%$ no investimento privado. Já o segundo conjunto de regressões estimado representa um painel estimado por efeito fixo e também por efeito aleatório, sem a inclusão de variáveis instrumentais, levando em consideração as interações criadas pelas dummies estaduais. Neste caso, a maioria dos estados apresentou um alto impacto do investimento público sobre o investimento privado, tendo uma elasticidade variando de $(0,74)$ a $(1,135)$. Alguns estados como Alagoas, Goiás, Mato Grosso, Pernambuco e Sergipe apresentaram um impacto relativamente baixo, quando comparados aos demais estados, pois o coeficiente estimado variou entre $(0,476)$ e $(0,668)$. Finalmente, o terceiro 
conjunto de regressões foi estimado das quatro maneiras distintas, levando em consideração as interações criadas pelas dummies regionais. Todas as macroregiões apresentaram um alto impacto do investimento público sobre o investimento privado, tendo um coeficiente variando entre $(0,896)$ e $(0,998)$. $\mathrm{O}$ único caso que apresentou um coeficiente baixo foi o da região Sul quando o painel sem variáveis instrumentais foi estimado por efeito fixo, sendo que o coeficiente resultante foi de $(0,656)$.

Desta forma, podemos concluir que há uma forte relação de complementaridade entre os dois tipos de investimento, o que por sua vez poderia favorecer a existência de algumas formas de parceria entre os setores público e privado de forma a melhorar o bem-estar social e também oferecer um retorno atraente aos investimentos realizados pelo setor privado.

Entendemos que a principal contribuição deste trabalho é mensurar o impacto do investimento público sobre o investimento privado. A partir dos resultados obtidos com as estimações, foi possível defender a existência de uma forte relação de complementaridade entre os dois investimentos. Entretanto, a análise efetuada restringe-se ao setor de construção civil, o qual está inteiramente relacionado à infra-estrutura urbana. Por isso, seria muito construtivo realizar uma análise sobre os investimentos em outros setores, de forma a verificar se essa forte relação de complementaridade entre os investimentos público e privado permanece.

\section{Referências Bibliográficas}

Aschauer, D. (1989), 'Does public capital crowd out private capital?', Journal of Monetary Economic 24, 171-188.

Bajo, O. \& Sosvilla, S. (1993), 'Does public capital affect private sector performance? an analysis of spanish case, 1964-1988', Economic Modelling 10, 179185.

Barros, C. M. (2005), Parceria público-privada: Um breve estudo sobre a experiência internacional recente, Technical report, CEPPEV-Centro de PósGraduação e Pesquisa Visconte de Cairu.

Easterly, W. \& Rebelo, S. (1993), 'Fiscal policy and economic growth: an empirical investigation', Journal of Monetary Economics 32, 418-458.

Ferreira, P. C. \& Malliagros, T. G. (1998), 'Impactos produtivos de infraestrutura no brasil: 1950-1995', Pesquisa e Planejamento Econômico 2.

Morandi, L. \& Reis, E. J. (2004), Estoque de capital fixo no brasil, 1950-2002, in 'Anais do XXXII Encontro Nacional de Economia - ANPEC'.

Nazmi, N. \& Ramirez, M. D. (1997), 'Public and private investment and economic growth in méxico', Contemporary Economic Policy 15.

Pinho, J. C. C. (2001), Impacto do investimento público na produção, in 'Congresso de Economia de Galícia: Novos Cenários para a Economia Galega'.

Ramirez, M. D. (1996), 'Public and private investment in méxico and chile: an empirical test of the complementarity hipothesis', Atlantic Economic Journal 24, 301-320. 
Rocha, C. H. \& Teixeira, J. R. (1996), 'Complementaridade versus substituição entre investimento público e privado na economia brasileira: 1965-90’, Revista Brasileira de Economia $\mathbf{5 0 .}$

Sant'Ana, T., Rocha, C. H. \& Teixeira, J. R. (1994), The impact of public investment on private capital formation in brazil: 1965-85, in 'Brazilian Meeting of Operational Research'.

Servén, L. (1996), Does public capital crowd out private capital? evidence from Índia, Technical report, World Bank Policy Research Working Paper Series.

Silveira, A. C. B. M. (2005), Determinantes dos investimentos privados: evidência empírica para os estados brasileiros na década de 1990, Technical report, FEA/USP.

Wooldridge, J. M. (2003), Introductory econometrics: A modern approach, Technical report, Michigan State University. 